\title{
Zinc sulphate administered by transdermal iontophoresis improves breaking strength of surgical wounds in skin of alloxan-induced diabetic rats ${ }^{1}$
}

\author{
Lucas Langoni Cassettari ${ }^{\mathrm{I}}$, Pedro Colli Rocha Dias ${ }^{\mathrm{II}}$, Amanda Natália Lucchesi ${ }^{\mathrm{II}}$, Maurício Ferraz de Arruda ${ }^{\mathrm{IV}}$, César Tadeu \\ Spadellav $^{\mathrm{v}}$
}

${ }^{\mathrm{I}} \mathrm{MS}$, Fellow PhD degree, Postgraduate Program in General Basis of Surgery, Faculty of Medicine, Sao Paulo State University (UNESP), Botucatu-SP, Brazil. Technical procedures, acquisition of data, manuscript writing.

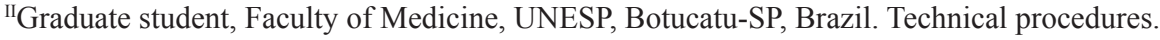

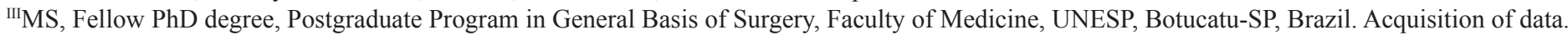
${ }^{\text {IV }} \mathrm{PhD}$, Assistant Professor, Physiotherapy Course, Municipal Institute of Higher Education, IMES-FAFICA, Catanduva-SP, Brazil. Orientation on the standardization of the method and application of transdermal iontophoresis.

${ }^{\mathrm{v}} \mathrm{PhD}$, Full Professor, Department of Surgery and Orthopedics, Faculty of Medicine, UNESP, Botucatu-SP, Brazil. Supervised all phases of the study, manuscript writing, critical revision, final approval of the version to be published.

\section{ABSTRACT}

PURPOSE: To investigate the effect of zinc sulphate administered by transdermal iontophoresis (TDI) on mechanical resistance of surgical wounds performed in the skin of diabetic rats.

METHODS: One hundred and sixty male Wistar rats weighing approximately $250 \mathrm{~g}$ were submitted to an incision surgery at the anterior region of abdomen and randomly distributed into four experimental groups with 40 non-diabetic control animals (G1) and 40 untreated diabetic animals (G2), both without any treatment of incisions; 40 non-diabetic animals (G3) and 40 untreated diabetic animals (G4), both with incisions treated with zinc sulphate, administered for a period of four consecutive days after surgery, in sessions of ten minutes duration, using a continuous-current electrostimulator ( $\mathrm{Zn}+\mathrm{TDI})$. Each experimental group was further divided into four subgroups with ten rats each to be evaluated on the 4th, 7th, 14th, and 21 st day after surgery. In each period were analyzed clinical and laboratory from the animals, and measured the breaking strength and hydroxyproline content (OH-P) of the skin scars.

RESULTS: Breaking strength (BS) was significantly reduced $(p<0.05)$ in skin scars of untreated diabetic rats $(\mathrm{G} 2)$ on the $7^{\text {th }}, 14^{\text {th }}$, and 21st postoperative days when compared to non-diabetic control rats (G1). In contrast, BS in skin scars of non-diabetic and untreated diabetic rats $(\mathrm{G} 3, \mathrm{G} 4)$ treated with $\mathrm{Zn}+$ TDI showed significant increase $(\mathrm{p}<0.05)$ in those periods when compared with their respective controls with untreated incisions. The OH-P content of the scars did not show statistically significant variation in all studied groups at four different times evaluated after surgery.

CONCLUSIONS: Zinc sulphate administered by transdermal iontophoresis had beneficial effect on the mechanical resistance of scars produced in the skin of diabetic rats. This therapeutic may have potential to reduce the complications observed in surgical wounds of the skin in diabetic subjects, mainly in most vulnerable stages of incisions to dehiscences, leakages and infections.

Key words: Iontophoresis. Zinc Sulfate. Diabetes mellitus. Wound Healing. Alloxan. Rats. 


\section{Introduction}

Diabetes mellitus (DM) is considered to be a public health problem worldwide, and it is associated by high morbidity and mortality rates ${ }^{1,2}$. Failures observed in the healing process of diabetic patients' wounds are the major source of complications associated with surgical procedures involving increased risk for dehiscences, leakages, and infections ${ }^{3,4}$.

DM causes impairment of immune cells, causing difficulties in the process of tissue repair, which occur in the initial stages of the healing process, where increased inflammation, decreased vascular proliferation and an altered function and properties of monocytes, macrophages, and fibroblasts are observed $^{5,6}$. Wound remodeling phase is also altered in DM, where the process of organization of the collagen deposited in the scars is defective ${ }^{7}$.

Long-term studies have shown that hyperglycemia control plays an important role in the genesis and development of chronic diabetic complications ${ }^{8}$, and it can also improve alterations in the tissue repair process. Additionally, emphasis has been given to the application of therapeutic resources that can enhance blood circulation in the wounded area, specifically in the skin ${ }^{9}$. Among such resources, the stimulation of tissues by continuous electric current, associated with ion administration (iontophoresis) has been widely used in clinical practice and in experimental studies, with several therapeutic purposes, including the localized calcium deposits, tendinitis and tenosynovitis, hyperhidrosis, myospasms, joint arthrosis, tissue adherence, keloids, rheumatoid arthritis, fungal infection, lymphedema and wound healing ${ }^{10,11}$.

Transdermal iontophoresis (TDI) is a non-invasive technique that uses a lower electric current potential than five volts, with current intensities oscillating between 0.1 and $1 \mathrm{~mA} / \mathrm{cm}^{2}$. It has the purpose to facilitate the transfer of ions and substances through the corneal layer of the skin in a controlled fashion.

Among the advantages of the technique, reduced risk infection and the possibility of administering the drug directly to the affected site, thus minimizing its adverse systemic effects, are noteworthy. TDI is also an alternative form to the injectable route; it is painless and less traumatic. The major ionizable substances used are acetic acid, adrenalin, sodium carbonate, calcium chloride, magnesium chloride, potassium iodate, ketoprofen, novocain, lidocaine, calcitonin, diclofenac, insulin and zinc sulphate.

Zinc, an orthomolecule that integrates insulin, acts on the metabolism of carbohydrates and collagen, and it is intrinsically related to the tissue repair process ${ }^{12}$. Postoperatively, serum levels of zinc may be reduced in diabetic animals and humans. Such deficiency can lead to reduced formation of connective tissue and change in tissue repair ${ }^{13}$.

Nevertheless, there are no clinical and experimental studies on the healing of surgical wounds in the skin, where the effects of TDI plus zinc have been evaluated. As experimental DM models can mimetize clinical, laboratory and histopathological changes of human diabetes, this work aims to check the actual potential of this therapy on the mechanical resistance and the tissue hydroxyproline content of these scars. We hope that our findings can contribute to a better therapeutic approach to chronic diabetic complications.

\section{Methods}

\section{Animals and diabetes induction}

Use of laboratory animals followed the ethical code for animal experimentation of the Council for International Organization of Medical Sciences (CIOMS), and was approved by the Animal Experimentation Ethics Committee of our institution. One hundred and sixty male Wistar rats, with approximately $250 \mathrm{~g}$, from the Central Animal Laboratory, Faculty of Medicine, UNESP, Botucatu-SP, were used in this experiment.

Animals were randomly assigned into four experimental groups, with 40 animals each, as follows: G1- Non-diabetic control rats without any treatment of the surgical incision; G2 - Untreated diabetic rats without any treatment of the surgical incision; G3 Non-diabetic rats with surgical incision treated with zinc sulphate administered by transdermal iontophoresis ( $\mathrm{Zn}+\mathrm{TDI})$; G4Untreated diabetic rats with surgical incision treated by $\mathrm{Zn}+$ TDI. Each experimental group was further divided into four subgroups with 10 animals each, to be evaluated and sacrificed, respectively, in four different times of the experiment, on the 4th, 7th, 14th and 21 st day after surgery.

Diabetes was induced by alloxan administered intravenously at a dose of $42 \mathrm{mg} / \mathrm{kg}$ of body weight using one of the tail veins. We only used animals with clinical signs of severe diabetes and fasting glucose $>250 \mathrm{mg} / \mathrm{dL}$ in two successive determinations (seven and 14 days after diabetes induction). Rats with mild diabetes or those who died throughout the experiment were replaced to avoid compromising the initial size of the sample.

\section{Analyzed parameters}

At the beginning of the experiment (14th day of followup or diabetes onset) and at each time point of follow-up, clinical 
(body weight, water intake, food intake and diuresis) and laboratory (blood glucose, urinary glucose, glycosylated hemoglobin and plasma insulin) parameters were analyzed. Clinical parameters were obtained using individual metabolic cages. Blood glucose was determined by standard enzymatic method (Johnson \& Johnson's), glycosylated hemoglobin by agarose gel electrophoresis (Sebia, France), and insulin by radioimmunoassay (Diagnostic Products Corporation, USA). At sacrifice, a segment of the skin containing the incision was removed from each rat to measure breaking strength (BS) of the scars. After performing the biomechanical assays representative fragments of the scars were also obtained for dosage of tissue OH-P using the Switzer method.

\section{Technical procedures}

All invasive procedures on animals were performed under general anesthesia using ketamine (100mg/kg of body weight) + xylazine $(50 \mathrm{mg} / \mathrm{kg}$ of body weight), administered intramuscularly (Rhobifarma Ind. Farmacêutica Ltda, Hortolândia-SP). Blood samples at sacrifice were obtained by cardiac puncture, with chest open. Euthanasia was by exsanguination, followed by the section of the vena cava infradiaphragmatic.

The surgical wounds were performed on the 15 th day of normal follow-up or of diabetes induction with the animals under 12-hour fasting. After general anesthesia and trichotomy of the anterior region of the abdomen, the rats were placed on a flat surface with their limbs extended, and antisepsis of the abdomen was performed by $2 \%$ iodinated. A median, medium-umbilical incision with $4 \mathrm{~cm}$ in length was performed with interest in the epidermis and dermis. The incision was immediately closed with 4-0 nylon strands (Ethicon Inc., USA) using simple separate stitches at a distance of $8 \mathrm{~mm}$ from each other.

To treat the surgical wounds with $\mathrm{Zn}+\mathrm{TDI}$, two electrodes were positioned on the corneal layer of the epidermis of each animal. The first was placed near the lower angle of the abdominal incision and the second in the interscapular dorsal region. Both electrodes were connected to a continuous-current electrostimulator (Ibramed, São Paulo, Brazil). The abdominal electrode was connected to the anode (positive) of the device, and that located on the dorsal region to the cathode (negative). The electric current emitted standardized amplitude of $2 \mathrm{~mA}$, with 10-minute treatment sessions. Each session was performed at four different times in the postoperative period, of which the first was immediately after the performance of the surgical incision (immediate PO) and the other on the three subsequent days (1st, 2nd and 3rd PO).
According to the proposed treatment, a small gauze pad moistened with $4 \mathrm{ml}$ of zinc sulphate solubilized at the concentration of $100 \mathrm{mg} / \mathrm{kg}$ of body weight was interposed between the electrode positioned on the base of the abdominal incision and the corneal layer of the epidermis for each experimental group, as shown in Figure 1.
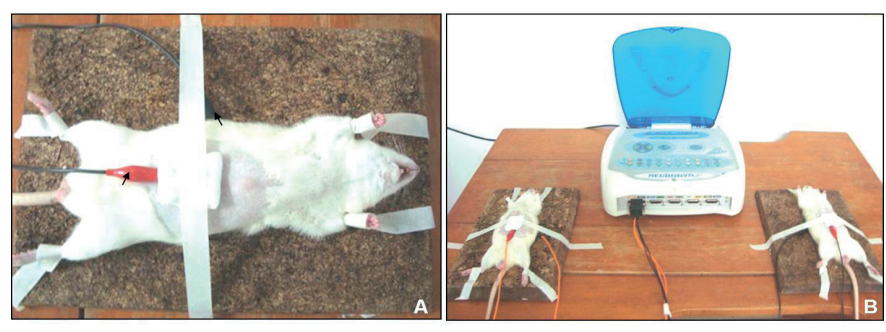

FIGURE 1 - Illustration of the preparation of animals for treatment by means of iontophoresis. A) Detail of the animal with electrodes fixed in the anterior region of the abdomen and on the dorsal region (arrows) for treatment commencement. B) Animals during the electrotherapeutic treatment.

\section{Breaking strength and hydroxyproline content measures}

To measure the breaking strength of scars, all sutures were removed and a rectangular segment of the skin, measuring $1.5 \mathrm{~cm}$ in length by $0.8 \mathrm{~cm}$ in width with the incision in the center, was carefully removed. Then, the segment was pulled at both ends by two claws until breaking by using a universal machine for biomechanical assays (EMIC, DL 10,000 model - Sao Paulo, Brazil). Figure 2 illustrates the sequence of procedures used.
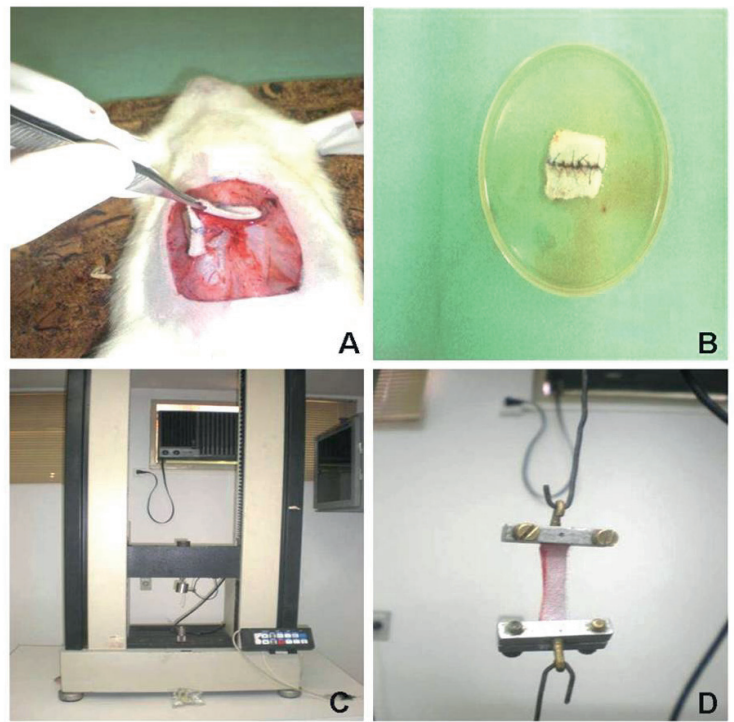

FIGURE 2 - Sequence of procedures used to measure the breaking strength of skin scars of the animals. A) Removing of a rectangular segment of skin containing the incision; B) Segment of skin removed with incision located in the center; C) Universal machine used for biomechanical assays; D) Skin segment without sutures being pulled at both ends by two claws until breaking. 


\section{Statistical analysis}

The study of the clinical, laboratory and biomechanical variables, according to the 4 experimental groups and the 4 sacrifice times, was conducted by means of $4 \times 4$ factorial analysis of variance, in a completely randomized design, complemented by the Tukey's multiple comparisons test for homogeneous or parametric variables or by Mann-Whitney and Kruskal-Wallis' non-parametric analysis for the variables showing results with a heterogeneous distribution. All the statistical discussions in the study were performed at the level of significance of $5 \%$ or $\mathrm{p}<0.05$.

\section{Results}

\section{Clinical and laboratory findings}

Non-diabetic rats (G1, G3) showed clinical and laboratory parameters that were compatible with those observed for normal animals of the same lineage in all periods of evaluation in the experiment. In contrast, untreated diabetic rats (G2, G4) without any treatment for the disease developed with accentuated body weight loss and significantly increased water intake, food intake and diuresis as compared with non-diabetic control animals $(p<0.001)$. The levels of blood glucose and glycosylated hemoglobin were also high in diabetic rats from the both groups, and the values for plasma insulin were significantly low $(\mathrm{p}<0.001)$. Figures 3 and 4 show the clinical and laboratory findings.
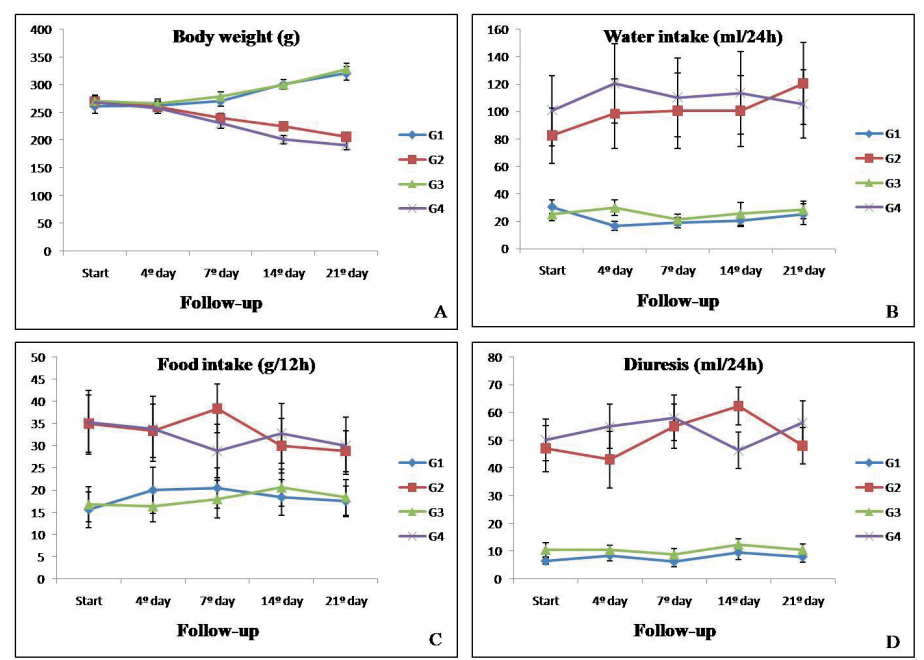

FIGURE 3 - Graphs showing the variation of clinical parameters in four experimental groups during follow-up. A) Body weight (g); B) Water intake (ml/24h); C) Food intake (g/12h); D) Diuresis (ml/24h).
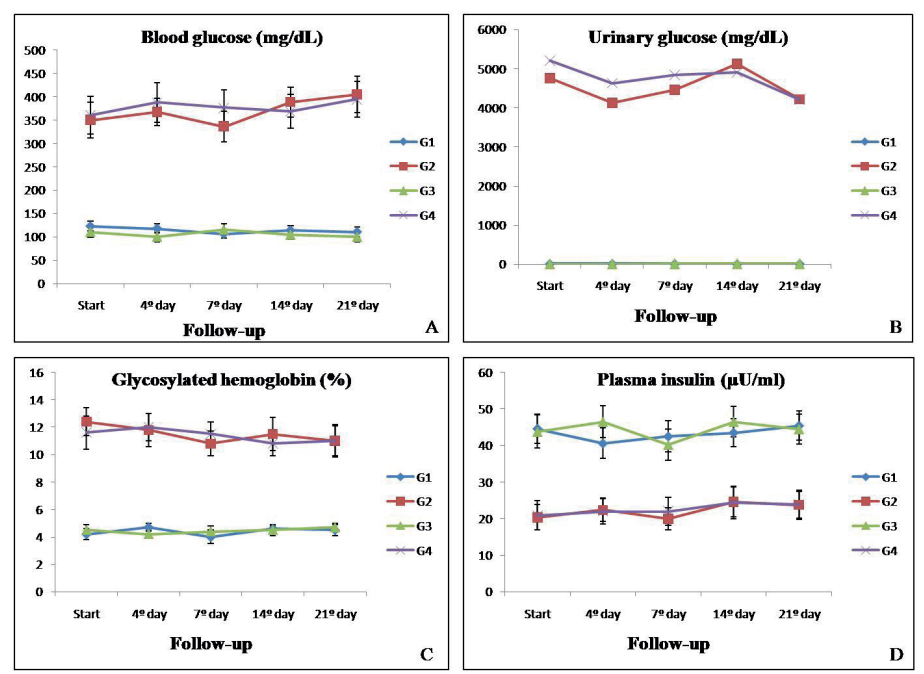

FIGURE 4 - Graphs showing the variation of laboratory parameters in four experimental groups during follow-up. A) Blood glucose (mg/dL); B) Urinary glucose (mg/dL); C) Glycosylated hemoglobin (\%); D) Plasm insulin $(\mu \mathrm{IU} / \mathrm{ml})$.

\section{Biomechanical assays and tissue OH-P content}

The breaking strength of scars in the skin of non-diabetic control and untreated diabetic rats, with or without treatment of the surgical incision with $\mathrm{Zn}+\mathrm{TDI}$, did not show statistically significant differences on the 4 th postoperative day.

However, untreated diabetic rats, with or without treatment of the surgical incision, showed significant lower breaking strength values than those observed for non-diabetic control rats $(\mathrm{p}<0.05)$, on the 7 th, 14 th and 21 st postoperative days. In turn, zinc sulphate, administered by TDI, significantly increased the breaking strength of surgical scars produced in the skin of non-diabetic control (G3) and untreated diabetic (G4) rats when compared to their respective controls without any incision treatment in the same periods (Table 1).

Despite the breaking strength variations, there were no significant differences in values of the tissue $\mathrm{OH}-\mathrm{P}$ content in the skin scars of non-diabetic or untreated diabetic rats at any time point of the postoperative follow-up. 
TABLE 1 - Means \pm SD of the breaking strength values (gf) of skin scars of rats from the four experimental groups at the four different times of sacrifice.

\begin{tabular}{ccccc}
\hline \multirow{2}{*}{ Groups } & \multicolumn{4}{c}{ Times of Sacrifice } \\
\cline { 2 - 5 } & $\mathbf{4}^{\mathbf{0}}$ day & $\mathbf{7}^{\mathbf{0}}$ day & $\mathbf{1 4}^{\mathbf{0}}$ day & $\mathbf{2 1}^{\mathbf{0}}$ day \\
\hline G1 & $5.96 \pm 2.93$ & $176.98 \pm 5.62$ & $304.16 \pm 5.38$ & $656.92 \pm 22.12$ \\
G2 & $4.34 \pm 2.16^{\mathrm{ns}}$ & $35.60 \pm 2.57^{*}$ & $95.52 \pm 2.23^{*}$ & $256.25 \pm 10.84^{*}$ \\
G3 & $6.36 \pm 2.40$ & $414.78 \pm 55.35$ & $682.30 \pm 67.42$ & $1678.40 \pm 151.12$ \\
G4 & $4.90 \pm 2.36^{\mathrm{ns}}$ & $189.41 \pm 22.65^{*}$ & $342.61 \pm 95.74^{*}$ & $805.26 \pm 62.35^{*}$ \\
\hline
\end{tabular}

${ }^{\mathrm{ns}} \mathrm{G} 1=\mathrm{G} 2=\mathrm{G} 3=\mathrm{G} 4$ on the 4 th day $(\mathrm{p}>0.05)$

${ }^{*} \mathrm{G} 2<\mathrm{G} 1$ on the 7 th, and 14 th days $(\mathrm{p}<0.05)$ and on the 21 st day $(\mathrm{p}<0.01) ; \mathrm{G} 2<\mathrm{G} 4$ on the 7th,14th, and 21st days ( $<<0.001)$; G4 $<\mathrm{G} 3$ on the 7 th, 14 th, and 21 st days $(\mathrm{p}<0.001)$

\section{Discussion}

Transdermal iontophoresis (TDI) is a technique used to improve the penetration of chemical compounds through skin using low-amperage continuous electric current ${ }^{14}$. Through the electroosmosis process, TDI increases the permeation of charged and neutral compounds and provides the option for distribution of programmed medication. Combinations of iontophoresis with chemistry, electroporation and sonophoresis intensifiers have been tested to increase the transdermal permeation of drugs and reducing possible adverse effects.

In this study, it was observed that diabetic rats showed significant reduction in the breaking strength of scars produced in the skin in the most vulnerable phases of the surgical incision when compared to non-diabetic control rats, which would predispose to greater incidence of dehiscences, fistulas and infections. Such alteration, in turn, was reversed by the use of TDI associated with zinc sulphate administration. Reduced mechanical resistance in incisions produced in the skin of diabetic animals has been described in the literature. While studying the mechanical properties of incision wounds in untreated diabetic rats seven, ten and 20 days after surgery, Andreassen and Oxlund ${ }^{15}$ observed that the animals showed reduced scar tensile strength for 20 days after surgery. Such alteration, however, has not been observed in insulin-treated diabetic animals, thus showing that hormone is essential for the adequate healing process of surgical wounds in the presence of diabetes. In their study on the influence of old age on the mechanical resistance of colonic anastomoses and wounds in the incision skin of diabetic rats, Seyer-Hansen et al..$^{16}$ concluded that the healing of colonic anastomoses in diabetic rats was not compromised by old age whereas the strength of skin wounds was reduced. In another study on the mechanical resistance in the skin of diabetic rats, Verhofstad and Hendriks ${ }^{17}$ reported that diabetes directly affects skin resistance, with reduced strength, that is also accompanied by reduced collagen content.

However, clinical or experimental studies in which the therapeutic potentialities of zinc administered by TDI have been tested are scarce. Cornwall ${ }^{18}$ evaluated the efficacy of TDI associated with zinc in a 71-year-old patient submitted to bilateral leg amputation who developed with ulcerations in the stumps. The treatment was performed for 20 days with 20-minute sessions. After that period, the wound healing areas were estimated, and, in total, almost $100 \%$ repair was obtained in relation to the initial findings. The therapeutic validity of the proposal, however, could not be confirmed due to the lack of subsequent studies.

In our study, the intensity of the electric current used was in accordance with the rule suggested by others ${ }^{19}$, who determined doses of 0.1 to $1 \mathrm{~mA}$ per area of the electrode in square centimeters $\left(\mathrm{cm}^{2}\right)$, and the ratio of 0.3 to $0.5 \mathrm{~mA} / \mathrm{cm}^{2}$ is the most frequently described in clinical studies using iontophoresis in humans. Nevertheless, since the size of the electrode must be compatible with the target area to be treated, occasionally, the current intensity used may reach values that predispose patients to risk for topical skin irritation and burns determined by the stimulation of free nerve terminations. The target electrode used in our study had $4 \mathrm{~cm}$ in height by $2 \mathrm{~cm}$ in width, that is, an area corresponding to $8 \mathrm{~cm}^{2}$. If the proposed method, preconizing $0.3 \mathrm{~mA} / \mathrm{cm}^{2}$, were used, the amplitude of $2.4 \mathrm{~mA}$ would be reached. Due to the milliamperage provided by the equipment, we preconized a dose of $2 \mathrm{~mA}$, which was not associated with any tissue damage in the animals' skin.

Despite the low values for breaking strength in the scars of diabetic animals in this study, the variation in the amount of tissue hydroxyproline present in these scars was not observed. This fact suggests that the healing defects observed in diabetic individuals may be much more dependent on the level of organization and maturation of the collagen deposited in the scars than, in fact, on its content.

Ortolan et $a .^{7}$ observed that, despite the inexistence of alterations in the amount of hydroxyproline present in anastomoses in the small and large intestines of alloxan-induced diabetic rats, the collagen deposited in the scars shows to be totally disorganized, little dense and composed of immature collagen fibers even in the later phases of the healing process. Similarly, these alterations may also be present in the incisions produced in the skin of the diabetic animals in this study, thus explaining the reduced mechanical resistance of their scars in the earlier healing phases. However, further studies are required to confirm the issues raised here. 


\section{Conclusion}

The topical application of zinc sulphate administered by transdermal iontophoresis in surgical incisions produced in the skin of untreated diabetic animals improves mechanical resistance in the scars in the most vulnerable phases of healing to dehiscences and infections without imposing risk or undesirable effects to the animals. These observations may open perspectives for the conduction of translational studies with the purpose to minimize healing defects of surgical wounds in diabetic patients.

\section{References}

1. American Diabetes Association. Diabetes Statistics. - data from the 2011 National Diabetes Fact Sheet [serial on the internet ]. [Access on 2013 March 2019]. Available from: http://www.diabetes.org/ diabetes-basics/diabetes-statistics/?loc=DropDownDB-stats.

2. World Health Organization (WHO). Health topics: Diabetes. [serial on the internet ]. [acess on 2013 March 19]. Available from: http:// www.who.int/topics/diabetes mellitus/en/

3. Rosenberg CS. Wound healing in the patient with diabetes mellitus. Nurs Clin North Am. 1990;25:247-53.

4. Nathan DM. Long-term complications of diabetes mellitus. N Engl J Med. 1993; 328: 1676-85.

5. Komesu MC, Tanga MB, Buttros KRm Nakao C. Effects of acute diabetes on rat cutaneous wound healing. Pathophysiology. 2004;11:63-7.

6. Carvalho PTC, Mazzer N, Reis FA, Belchior ACG, Silva IS. Analysis of the influence of low-power HeNe laser on the healing of skin wounds in diabetic and non-diabetic rats. Acta Cir Bras. 2006;21:177-83.

7. Ortolan EVP, Spadella CT, Caramori C, Machado JLM, Gregório EA, Rabello K. Microscopic, morphometric and ultrastructural analysis of anastomotic healing in the intestine of normal and diabetic rats. Exp Clin Endocrinol Diabetes. 2008;116:198-202.

8. The Diabetes Control and Complications Trial Research Group. The effect of intensive treatment of diabetes on the development and progression of long-term complications in insulin-dependent diabetes mellitus. N Engl J Med. 1993;14:977-86.

9. Nand A, Nand S, Ghilzai NM. Current developments using emerging transdermal techonologies in physical enhancement methods. Curr Drug Deliv. 2006;3:233-42.

10. Carvalho DC, Gonçalves RAA. Iontoforese e seu mecanismo de ação com o tecido humano. Lato and Sensu. 2003;4:3-5.

11. Dixit N, Bali V, Baboota S, Ahuja A, Ali J. Iontophoresis - an approach for controlled drug delivery: a review. Curr Drug Deliv. 2007;4(1):1-10.

12. Lansdown ABG. Zinc in the healing wound. Lancet. 1996;347:7067.

13. Moraes SP, Chaves FRB, Banci S, Rover PA, Georgetti F, Reis-Net JÁ. Zinco e cromo na cicatrização de feridas em ratos normais e diabéticos. Rev Col Bras Cir. 2000;27:6.

14. Batheja P, Thakur R, Michiniak B. Transdermal iontophoresis. Expert Opin Drug Deliv. 2006;3:127-38.

15. Andreassen TT, Oxlund $H$. The influence of experimental diabetes and insulin treatments on the biochemical properties of rat skin incisional wounds. Acta Chir Scand. 1987;153:405-9.

16. Seyer-Hansen M, Andreassen TT, Oxlund H. Strength of colonic anastomoses and skin incisional wounds in old rats - influence by diabetes and growth hormone. Growth Horm IGF Res. 1999;9:25461.

17. Verhofstadt MHJ, Hendriks TH. Diabetes impairs the development of early strength, but not the accumulation of collagen, during intestinal anastomotic healing in the rat. Br J Surg. 1994;81:1040-5.

18. Cornwall MW. Zinc iontophoresis to treat ischemic skin ulcers. Phys Ther. 1981;61:359-60.

19. Oliveira AS, Guaratini I, Castro MI. Fundamentação teórica para iontoforese. Rev Bras Fisioter. 2005;9:1-7.

\section{Acknowledgements}

To Patricia Pintor Reis for reviewing the text translated into English and Érika Veruska Ortolan for their valuable suggestions.

\section{Correspondence:}

César Tadeu Spadella

Faculdade de Medicina de Botucatu, Departamento de Cirurgia e Ortopedia

Campus da UNESP

Distrito de Rubião Jr., s/nº - Anexo Verde

18618-970 Botucatu - SP Brasil

Tel.: (55 14)3880-1447

spadella@fmb.unesp.br

Received: April 15, 2013

Review: June 17, 2013

Accepted: July 18, 2013

Conflict of interest: none

Financial sources: National Council of Technological and Scientific Development $(\mathrm{CNPq})$ and Coordination of Improvement for Higher Academic Staff (CAPES)

${ }^{1}$ Research performed at Experimental Surgery Laboratory, Faculty of Medicine, Sao Paulo State University (UNESP), Botucatu-SP, Brazil. Part of Master degree thesis, Postgraduate Program in General Basis of Surgery. Tutor: César Tadeu Spadella. 